OPEN ACCESS

Edited by:

Junjie $\mathrm{He}$,

Charles University, Czechia

Reviewed by:

Zhimin Wu,

Chongqing Normal University, China Minquan Kuang,

Southwest University, China

*Correspondence:

Kai Wang

wangkai@hrbmu.edu.cn

Li Zhang

lizhang@ccit.edu.cn

tThese authors have contributed equally to this work

Specialty section: This article was submitted to Theoretical and Computational Chemistry, a section of the journal Frontiers in Chemistry

Received: 05 October 2021 Accepted: 18 October 2021 Published: 15 November 2021

Citation:

Zhang L, Fang F, Cheng L, Lin H and Wang K (2021) Obvious Surface States Connecting to the Projected Triple Points in NaCl's

Phonon Dispersion.

Front. Chem. 9:789522.

doi: 10.3389/fchem.2021.789522

\section{Obvious Surface States Connecting to the Projected Triple Points in NaCl's Phonon Dispersion}

\author{
Li Zhang ${ }^{1 * \dagger}$, Fang Fang ${ }^{2 \dagger}$, Lixin Cheng ${ }^{2}$, Huiming Lin $^{3}$ and Kai Wang ${ }^{2 *}$ \\ $1^{1}$ College of Mechanics, Changchun Institute of Technology, Changchun, China, ${ }^{2}$ Engineering and Technology Center, The \\ Fourth Medical College of Harbin Medical University, Harbin, China, ${ }^{3}$ School of Chemistry, Harbin Normal University, Harbin, \\ China
}

With the development of computer technology and theoretical chemistry, the speed and accuracy of first-principles calculations have significantly improved. Using first-principles calculations to predict new topological materials is a hot research topic in theoretical and computational chemistry. In this work, we focus on a well-known material, sodium chloride $(\mathrm{NaCl})$, and propose that the triple point (TP), quadratic contact triple point (QCTP), linear and quadratic nodal lines can be found in the phonon dispersion of $\mathrm{NaCl}$ with $\mathrm{Fm} \overline{3} \mathrm{~m}$ type structure. More importantly, we propose that the clear surface states connected to the projected TP and QCTP are visible on the (001) surface. It is hoped that further experimental investigation and verification for these properties as mentioned above.

Keywords: DFT, first-principles calculations, phonon dispersion, surface state, $\mathrm{NaCl}$

\section{INTRODUCTION}

The recent rapid development in topological materials (Kong and Cui, 2011; Cava et al., 2013; Banik et al., 2018; Kumar et al., 2020; Li and Wei, 2021) makes chemists expect these materials to solve the current challenges in quantum chemistry. A series of topological materials, including topological insulators (Müchler et al., 2012; Bradlyn et al., 2017; Kou et al., 2017; Martín Pendás et al., 2019; Isaeva and Ruck, 2020), spin-gapless semiconductors (Gao et al., 2016; Wang et al., 2016; Wang, 2017; Sun et al., 2020; Yue et al., 2020), and topological semimetals/metals (Zhou et al., 2018a; Schoop et al., 2018; Xu et al., 2020a; Klemenz et al., 2020; Zhao et al., 2020), were predicted by researchers, and some of them are confirmed in experiments. Among them, topological semimetals/metals (Zhong et al., 2016; Zhang et al., 2018; Jin et al., 2019a; Jin et al., 2019b; He et al., 2019; Wang et al., 2020a; Wang et al., 2020b; Xu et al., 2020b; Guo et al., 2020; Jin et al., 2021) always have nontrivial band crossings in their electronic band structures. In addition to their potential applications in technology, they also provide a platform for the study of basic quasiparticles in low cost experiments.

Recently, parallel to electrons, topological concepts have been extended to boson systems such as phonons in crystal materials, classical elastic waves in macroscopic artificial phonon crystals, and magnetic oscillators in magnets. Especially important is that the topological phonon in crystal materials (Jin et al., 2018; Liu et al., 2019; Zheng et al., 2019; Liu et al., 2020; Xie et al., 2021) can provide a potential prospect for regulating heat transfer and electron-phonon interaction. It should be emphasized that the phonon is not limited by the principle of Pauli incompatibility, which means that the experimental detection can be carried out in the whole frequency region of the phonon spectrum.

This work will focus on a famous realistic material, sodium chloride $(\mathrm{NaCl}) . \mathrm{NaCl}$ is with the $\mathrm{Fm} \overline{3} \mathrm{~m}$ type cubic structure and with the space group number 225 . The experimental lattice constants 


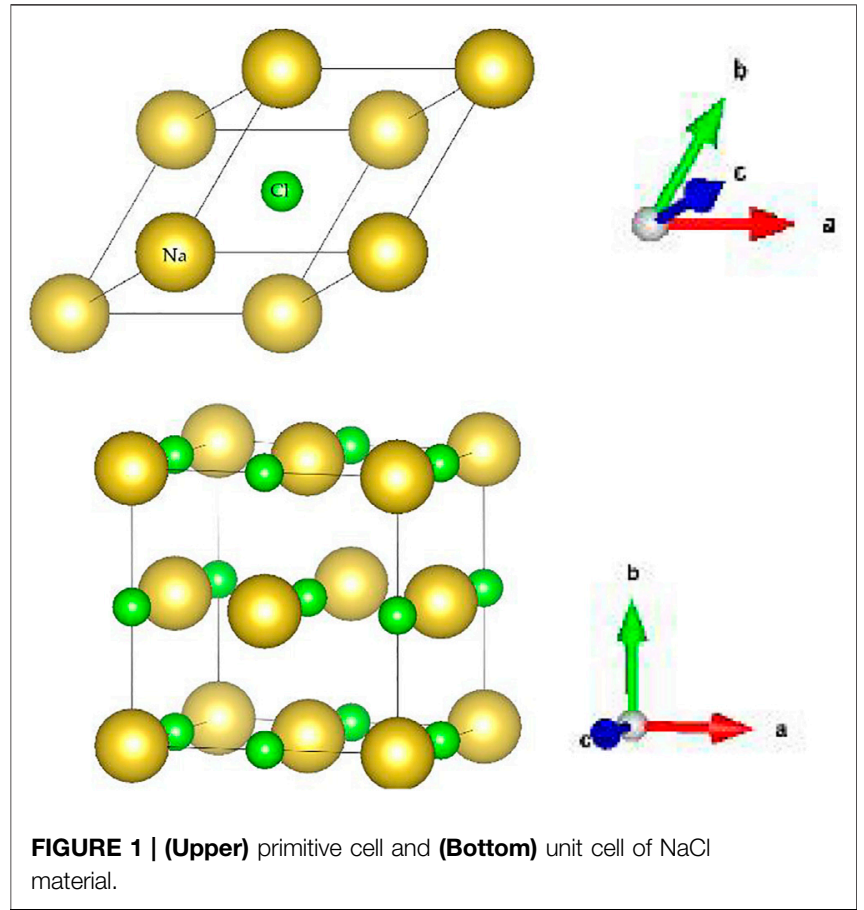

of sodium chloride (Abrahams and Bernstein, 1965) are $a=b=c=$ $5.62 \AA$. The Na locates at $4 \mathrm{a}(0,0,0)$ Wyckoff position, and the $\mathrm{Cl}$ locates at $4 \mathrm{~b}(0.5,0.5,0.5)$ Wyckoff position. In this work, using the first principles calculations, we will study the topological signatures of the NaCl's phonon dispersion. We found that triple points with linear phonon bands dispersion and quadratic phonon bands dispersion coexist in NaCl's phonon dispersion. More importantly, we will exhibit the interesting phonon surface states of the (001) plane. The authors hoped that the uncovered triple points and their connected surface states in the $\mathrm{NaCl}$ phonon system could be confirmed in experiment soon.

\section{METHODS}

The crystal structure of $\mathrm{Fm} \overline{3} \mathrm{~m} \mathrm{NaCl}$ is selected from the Materials Project database (Materials Project, 2021). Some material information, including the magnetic ordering, final magnetic moment, formation energy/atom, band structure, and the phonon dispersion of $\mathrm{NaCl}$, can be found in ref. (Materials Project, 2021). One concludes from ref. (Materials Project, 2021) that $\mathrm{NaCl}$ is a nonmagnetic semiconductor with a band-gap value of $5.145 \mathrm{eV}$. The obtained lattice constants based on first-principle calculations are $a=b=c=5.6916 \AA$, consisting well with the experiment values (Abrahams and Bernstein, 1965). The primitive cell and the unit cell of the $\mathrm{NaCl}$ are shown in Figure 1. The yellow and green balls represent the $\mathrm{Na}$ and $\mathrm{Cl}$ atoms, respectively. This work will focus on the phonon dispersion of $\mathrm{NaCl}$ because we would like to uncover its topological signatures. The phonon dispersion of $\mathrm{NaCl}$ is determine based on the density functional perturbation theory with the PHONOPY codes (Togo and Tanaka, 2015), and the topological surface properties are constructed by the WANNIERTOOLS package (Wu et al., 2018) based on the phononic Wannier tight-binding Hamiltonian.

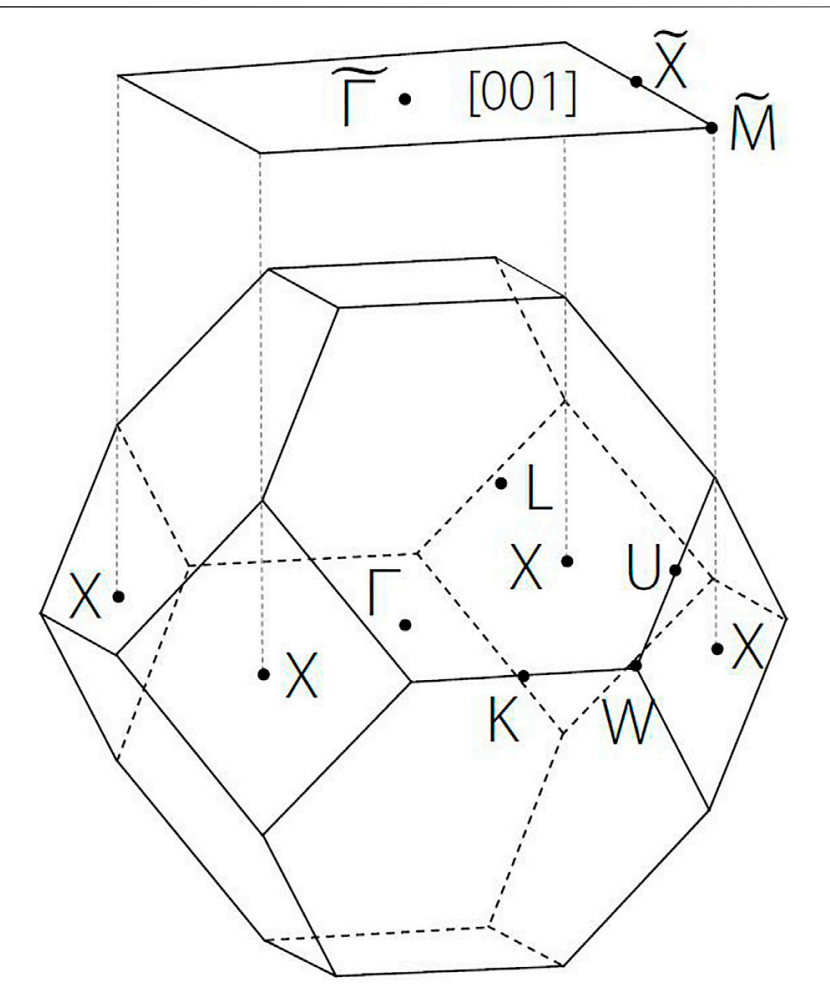

FIGURE 2 | Three-dimensional Brillouin zone (BZ) and the twodimensional (001) surface BZ. The X, K, W, Y, L, $\Gamma$ are the symmetry points of 3D BZ. $\Gamma, X$, and $X$ points are projected to $\widetilde{\boldsymbol{\Gamma}}, \tilde{\mathbf{X}}$, and $\tilde{\mathbf{M}}$ points of the (001) surface.

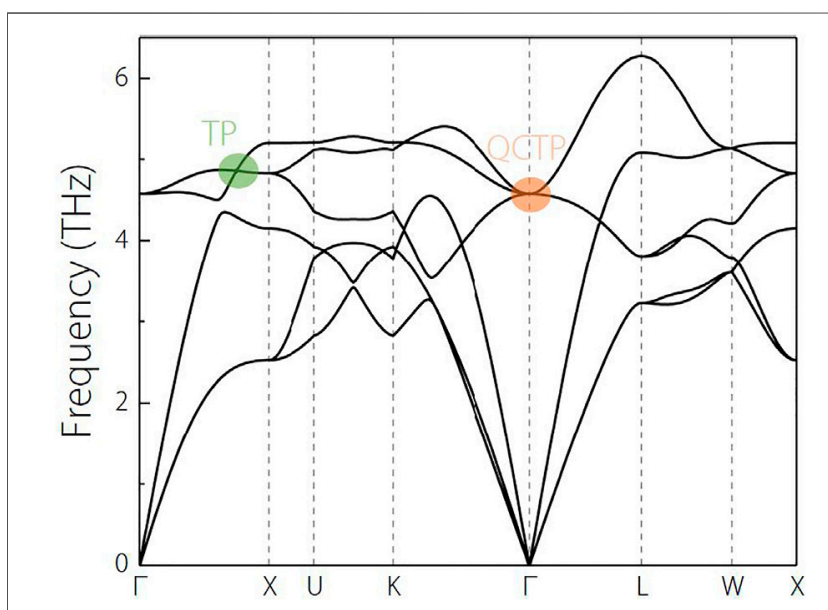

FIGURE 3 | Phonon dispersion of $\mathrm{NaCl}$ along the $\Gamma-X-U-K-\Gamma-L-W-X$ paths.

\section{Calculated Phonon Dispersion and the Related Topological Signatures}

In Figure 2, we plotted the three-dimensional BZ and some high symmetry points, $\mathrm{X}, \mathrm{K}, \mathrm{W}, \mathrm{Y}, \mathrm{L}$, and $\Gamma$. Along the $\Gamma-\mathrm{X}-\mathrm{U}-\mathrm{K}-\Gamma-\mathrm{L}-$ $\mathrm{W}$-X paths, the phonon dispersion of $\mathrm{NaCl}$ is calculated, and the results are shown in Figure 3. During the phonon dispersion 
A

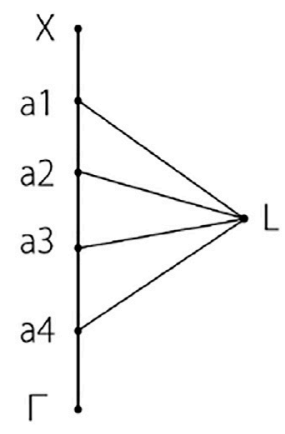

C

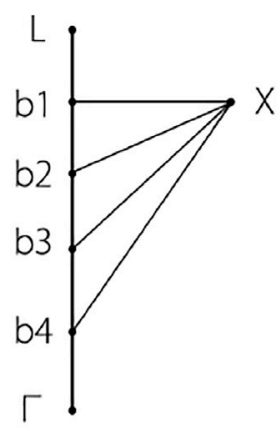

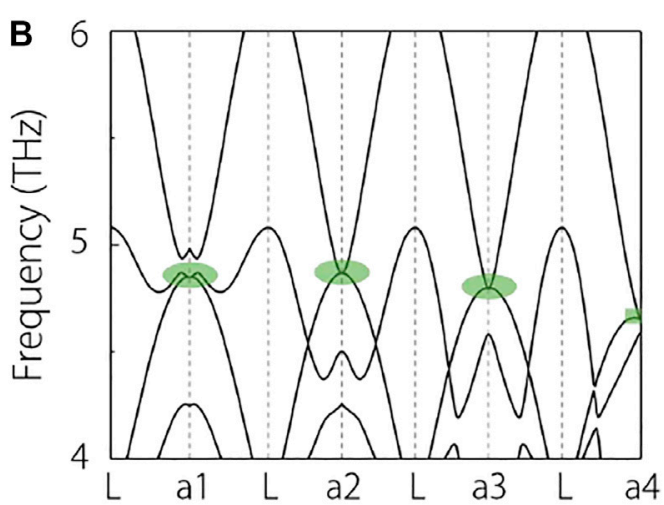

D

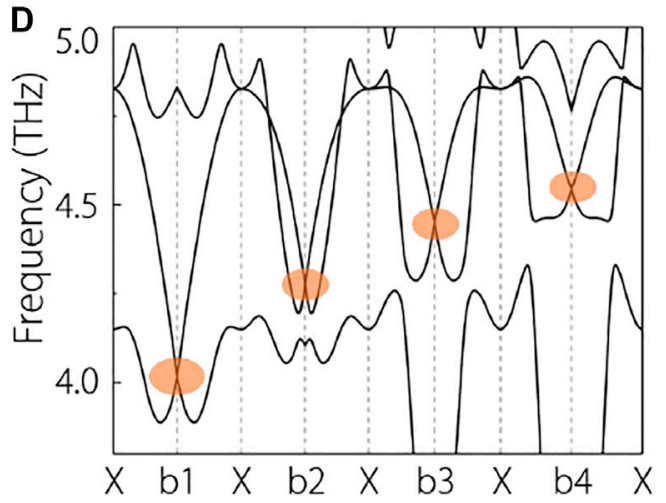

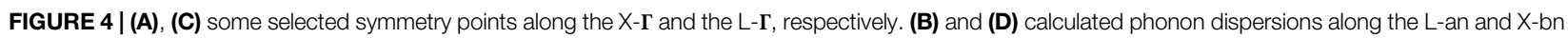
$(n=1-4)$. The linear two-degenerate points and the quadratic two-degenerate points are highlighted with orange and green circles, respectively.

calculations, we built a $2 \times 2 \times 2$ supercell for the $\mathrm{NaCl}$ system. From Figure 3, at first glance, one concludes that the $\mathrm{NaCl}$ is dynamically stable because the $\mathrm{NaCl}$ system has no imaginary frequencies.

Moreover, from Figure 3, one obtains the following information: 1) Along the $\Gamma-\mathrm{X}$ path and in the range of 4-5 THz frequencies, there are one doubly-degenerate phonon band and a non-degenerate phonon band, and these two bands cross at a point (see the green circle in Figure 3) along the $\Gamma-\mathrm{X}$ path. This point along the $\Gamma-X$ path is a triple point; 2) along the $\mathrm{K}-\Gamma$ path and in the range of $4-5 \mathrm{THz}$ frequencies, one concludes that three phonon bands touched at the $\Gamma$ point, forming a triple point (see the orange circle region in Figure 3). However, we would like to point out that the triple point on the $\Gamma-\mathrm{X}$ and at $\Gamma$ are different because the point on the $\Gamma-\mathrm{X}$ is with linear band dispersion and the point at the $\Gamma$ is with a quadratic band dispersion. Hence, the triple point on the $\Gamma-\mathrm{X}$ is called triple point (TP) (Zhu et al., 2016; Tian et al., 2021), and the triple point at the $\Gamma$ is usually called quadratic contact triple point (QCTP) (Hu et al., 2019). QCTP features a quadratic band splitting along any direction in momentum space. Along the $\Gamma-$ $\mathrm{L}$ path, one can see that there are also a doubly degenerate band and a non-degenerate band in the range of $4.5-6 \mathrm{THz}$ frequencies.

One may wonder whether the doubly degenerate band along the $\Gamma-\mathrm{X}$ (around the TP) and the $\Gamma-\mathrm{L}$ (around the QCTP) paths

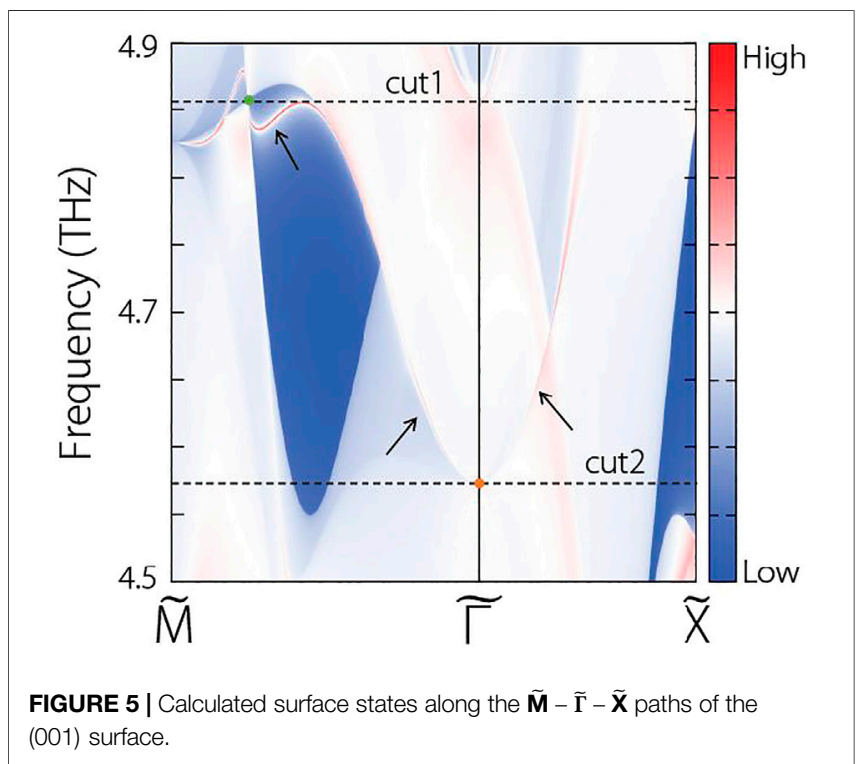

are the same. In the following, we will answer this question affirmatively. To better answer this question, in Figure 4A,C, we divided the $\Gamma-\mathrm{X}$ (around the TP) and $\Gamma-\mathrm{L}$ (around the QCTP) paths into five parts and selected some more symmetry points. Namely, we selected a1-a4 along the X- $\Gamma$ and b1-b4 along the L- $\Gamma$ 

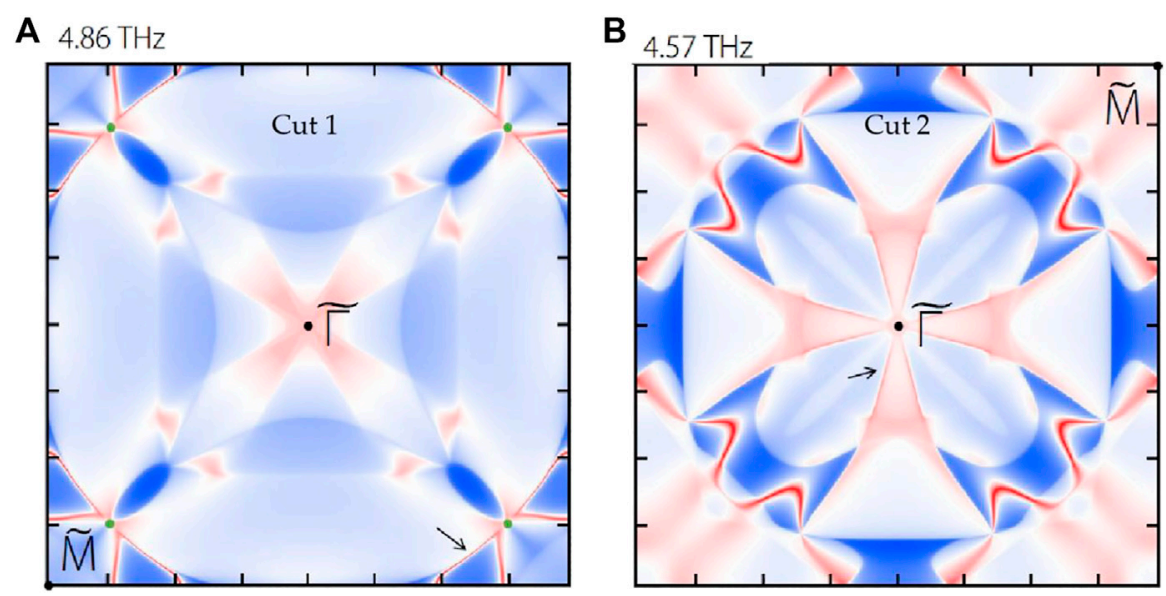

FIGURE 6 | Calculated iso-frequency surface contours at (A) $4.86 \mathrm{THz}$ and (B) $4.57 \mathrm{THz}$.

paths, respectively. The phonon dispersions along the L-an and X-bn $(n=1,2,3,4)$ are shown in Figure 4B,D, respectively. One finds the points at a1, a2, a3 a4 are all with a quadratic band splitting, however, for the points b1, b2, b3, b4, they are with a classic linear band splitting. Hence, the doubly degenerate band along the $\mathrm{X}-\Gamma$, is composed of doubly degenerate points with linear band splitting, forming a linear nodal line (Zhou et al., 2018b; Chen et al., 2018; Chang et al., 2019; Yan et al., 2019; Li et al., 2020a; Kirby et al., 2020; Meng et al., 2020; Wang and Yang, 2021). The doubly degenerate band along the $\Gamma-\mathrm{L}$, is composed of doubly degenerate points with quadratic band splitting, forming a quadratic nodal line (Yu et al., 2019; Wang et al., 2020c).

A summary of this section is shown as follow: $\mathrm{NaCl}$ phonon hosts a QCTP at the $\Gamma$ point, a TP along the X- $\Gamma$ path, a twodegenerate linear nodal line along the $\mathrm{X}-\Gamma$ path, and a quadratic nodal line along the $\Gamma-\mathrm{L}$. It is hoped that such rich topological signatures in $\mathrm{NaCl}$ can be confirmed in experiment soon.

\section{Calculated Surface States on (001) Surface BZ}

In this section, we come to study the project surface states of the [001] $\mathrm{NaCl}$ phonons. As shown in Figure 2, we selected some symmetry points, $\Gamma, X$ and $X$, and projected these points to $\widetilde{\Gamma}, \widetilde{X}$, and $\widetilde{\mathbf{M}}$ points of the (001) surface. In Figure 5, we collected the results and labeled the positions of the projected TP (green dot) and the projected QCTP (orange dot). One concludes that prominent surface states (Xu et al., 2015; Morali et al., 2019; Li et al., 2020b) connected to the projected $\mathrm{TP}$, which is benefit for experimental detection. Although the surface state connected to the QCTP is a little fuzzy, we can observe its trend and general shape.

For clarity, we also exhibit the iso-frequency surface contours at $4.86 \mathrm{THz}$ and $4.57 \mathrm{THz}$ in Figure 6A,B, respectively. In Figure 6A, the positions of the projected TP and the connected surface states are marked by a green dot and black arrows, respectively. In Figure 6B, the positions of the projected QCTP and the connected surface states are marked by a black dot and black arrows, respectively. The projected TP/QCTP connected surface states are visible.

\section{SUMMARY}

In this study, we proposed the topological signatures of the $\mathrm{NaCl}$ 's phonon dispersion. A systematic theoretical investigation found that this material hosts quadratic and linear nodal lines, TP and QCTP in its phonon dispersion. The QCTP is located at the $\Gamma$ position, the TP is along the $\mathrm{X}-\Gamma$, the linear nodal line is along the $\mathrm{X}-\Gamma$ path, and the quadratic nodal line is along the $\Gamma-\mathrm{L}$. Besides, the surface states are computed and clear surface arc states connected to the projected TP and QCTP can be observed on the (001) surface. Further experimental investigation and verification for these rich topological signatures are expected.

\section{DATA AVAILABILITY STATEMENT}

The original contributions presented in the study are included in the article/Supplementary Material, further inquiries can be directed to the corresponding authors.

\section{AUTHOR CONTRIBUTIONS}

LZ, and FF: conceptualization, methodology, software, formal analysis, data curation, and writing. KW, LC, HL, and LZ: investigation, funding, and project administration. All authors contributed to the article and approved the submitted version.

\section{FUNDING}

This work is supported by Topic Foundation of Changchun Institute of Technology (Grant No. 320200040), Young People Foundation of Changchun Institute of Technology 
(Grant No. 320200033), Doctor Foundation of Changchun Institute of Technology 2021, Natural Science Foundation of Heilongjiang Province (Grant No. LH 2020H067), Heilongjiang Postdoctoral Program (LBH-Q16173), Science

\section{REFERENCES}

Abrahams, S. C., and Bernstein, J. L. (1965). Accuracy of an Automatic Diffractometer. Measurement of the Sodium Chloride Structure Factors. Acta Crystallogr. 18 (5), 926-932. doi:10.1107/s0365110x65002244

Banik, A., Roychowdhury, S., and Biswas, K. (2018). The Journey of Tin Chalcogenides towards High-Performance Thermoelectrics and Topological Materials. Chem. Commun. 54 (50), 6573-6590. doi:10.1039/ c8cc02230e

Bradlyn, B., Elcoro, L., Cano, J., Vergniory, M. G., Wang, Z., Felser, C., et al. (2017). Topological Quantum Chemistry. Nature 547 (7663), 298-305. doi:10.1038/ nature 23268

Cava, R. J., Ji, H., Fuccillo, M. K., Gibson, Q. D., and Hor, Y. S. (2013). Crystal Structure and Chemistry of Topological Insulators. J. Mater. Chem. C 1 (19), 3176-3189. doi:10.1039/c3tc30186a

Chang, T. R., Pletikosic, I., Kong, T., Bian, G., Huang, A., Denlinger, J., et al. (2019). Realization of a Type-II Nodal-Line Semimetal in $\mathrm{Mg}_{3} \mathrm{Bi}_{2}$. Adv. Sci. 6 (4), 1800897. doi:10.1002/advs.201800897

Chen, H., Zhang, S., Jiang, W., Zhang, C., Guo, H., Liu, Z., et al. (2018). Prediction of Two-Dimensional Nodal-Line Semimetals in a Carbon Nitride Covalent Network. J. Mater. Chem. A 6 (24), 11252-11259. doi:10.1039/c8ta02555j

Gao, G., Ding, G., Li, J., Yao, K., Wu, M., and Qian, M. (2016). Monolayer MXenes: Promising Half-Metals and Spin Gapless Semiconductors. Nanoscale 8 (16), 8986-8994. doi:10.1039/c6nr01333c

Guo, C., Zhao, B., Huang, D., and Fan, S. (2020). Radiative thermal Router Based on Tunable Magnetic Weyl Semimetals. ACS Photon. 7 (11), 3257-3263. doi:10.1021/acsphotonics.0c01376

He, T., Zhang, X., Meng, W., Jin, L., Dai, X., and Liu, G. (2019). Topological Nodal Lines and Nodal Points in the Antiferromagnetic Material $\beta-\mathrm{Fe}_{2} \mathrm{PO}_{5}$. J. Mater. Chem. C 7 (40), 12657-12663. doi:10.1039/c9tc04046c

Hu, J., Wu, W., Zhong, C., Liu, N., Ouyang, C., Yang, H. Y., et al. (2019). Threedimensional Honeycomb Carbon: Junction Line Distortion and Novel Emergent Fermions. Carbon 141, 417-426. doi:10.1016/j.carbon.2018.09.027

Isaeva, A., and Ruck, M. (2020). Crystal Chemistry and Bonding Patterns of Bismuth-Based Topological Insulators. Inorg. Chem. 59 (6), 3437-3451. doi:10.1021/acs.inorgchem.9b03461

Jin, L., Wang, L., Zhang, X., Liu, Y., Dai, X., Gao, H., et al. (2021). Fully SpinPolarized Weyl Fermions and In/out-Of-Plane Quantum Anomalous Hall Effects in a Two-Dimensional D0 Ferromagnet. Nanoscale 13 (11), 5901-5909. doi:10.1039/d0nr07556f

Jin, L., Zhang, X., Dai, X., Liu, H., Chen, G., and Liu, G. (2019). Centrosymmetric Li ${ }_{2} \mathrm{NaN}$ : a superior Topological Electronic Material with Critical-type Triply Degenerate Nodal Points. J. Mater. Chem. C 7 (5), 1316-1320. doi:10.1039/c8tc05930f

Jin, L., Zhang, X., He, T., Meng, W., Dai, X., and Liu, G. (2019). Topological Nodal Line State in Superconducting NaAlSi Compound. J. Mater. Chem. C 7 (34), 10694-10699. doi:10.1039/c9tc03464a

Jin, Y., Wang, R., and Xu, H. (2018). Recipe for Dirac Phonon States with a Quantized valley berry Phase in Two-Dimensional Hexagonal Lattices. Nano Lett. 18 (12), 7755-7760. doi:10.1021/acs.nanolett.8b03492

Kirby, R. J., Ferrenti, A., Weinberg, C., Klemenz, S., Oudah, M., Lei, S., et al. (2020). Transient Drude Response Dominates Near-Infrared Pump-Probe Reflectivity in Nodal-Line Semimetals ZrSiS and ZrSiSe. J. Phys. Chem. Lett. 11 (15), 6105-6111. doi:10.1021/acs.jpclett.0c01377

Klemenz, S., Hay, A. K., Teicher, S. M. L., Topp, A., Cano, J., and Schoop, L. M. (2020). The Role of Delocalized Chemical Bonding in Square-Net-Based Topological Semimetals. J. Am. Chem. Soc. 142 (13), 6350-6359. doi:10.1021/jacs.0c01227 and Technology Program of Academy of Medical Sciences of Heilongjiang Province (Grant No. 201805), Research Foundation of Education Bureau of Jilin Province (Grant No. JJKH20210666KJ).

Kong, D., and Cui, Y. (2011). Opportunities in Chemistry and Materials Science for Topological Insulators and Their Nanostructures. Nat. Chem. 3 (11), 845-849. doi:10.1038/nchem.1171

Kou, L., Ma, Y., Sun, Z., Heine, T., and Chen, C. (2017). Two-dimensional Topological Insulators: Progress and Prospects. J. Phys. Chem. Lett. 8 (8), 1905-1919. doi:10.1021/acs.jpclett.7b00222

Kumar, N., Guin, S. N., Manna, K., Shekhar, C., and Felser, C. (2020). Topological Quantum Materials from the Viewpoint of Chemistry. Chem. Rev. 121 (5), 2780-2815. doi:10.1021/acs.chemrev.0c00732

Li, C. Z., Wang, A. Q., Li, C., Zheng, W. Z., Brinkman, A., Yu, D. P., et al. (2020). Fermi-arc Supercurrent Oscillations in Dirac Semimetal Josephson Junctions. Nat. Commun. 11 (1), 1150-1157. doi:10.1038/s41467-020-15010-8

Li, Y., Xia, J., and Srivastava, V. (2020). The Tetragonal Monoxide of Platinum: a New Platform for Investigating Nodal-Line and Nodal-point Semimetallic Behavior. Front. Chem. 8, 704. doi:10.3389/fchem.2020.00704

Li, Z., and Wei, B. (2021). Topological Materials and Topologically Engineered Materials: Properties, Synthesis, and Applications for Energy Conversion and Storage. J. Mater. Chem. A 9 (3), 1297-1313. doi:10.1039/d0ta11072h

Liu, Q.-B., Fu, H.-H., Xu, G., Yu, R., and Wu, R. (2019). Categories of Phononic Topological Weyl Open Nodal Lines and a Potential Material Candidate: Rb2Sn2O3. J. Phys. Chem. Lett. 10 (14), 4045-4050. doi:10.1021/ acs.jpclett.9b01159

Liu, Q. B., Qian, Y., Fu, H. H., and Wang, Z. (2020). Symmetry-enforced Weyl Phonons. npj Comput. Mater. 6 (1), 1-6. doi:10.1038/s41524-020-00358-8

Martín Pendás, A., Contreras-García, J., Pinilla, F., Mella, J. D., Cardenas, C., and Muñoz, F. (2019). A Chemical Theory of Topological Insulators. Chem. Commun. 55 (82), 12281-12287. doi:10.1039/c9cc04054d

Meng, W., Liu, Y., Zhang, X., Dai, X., and Liu, G. (2020). A NonsymmorphicSymmetry-Protected Hourglass Weyl Node, Hybrid Weyl Node, Nodal Surface, and Dirac Nodal Line in $\mathrm{Pd}_{4} \mathrm{X}(\mathrm{X}=\mathrm{S}, \mathrm{Se})$ Compounds. Phys. Chem. Chem. Phys. 22 (39), 22399-22407. doi:10.1039/d0cp03686b

Morali, N., Batabyal, R., Nag, P. K., Liu, E., Xu, Q., Sun, Y., et al. (2019). Fermi-arc Diversity on Surface Terminations of the Magnetic Weyl Semimetal $\mathrm{Co}_{3} \mathrm{Sn}_{2} \mathrm{~S}_{2}$. Science 365 (6459), 1286-1291. doi:10.1126/science.aav2334

Müchler, L., Zhang, H., Chadov, S., Yan, B., Casper, F., Kübler, J., et al. (2012). Topological Insulators from a Chemist's Perspective. Angew. Chem. Int. Ed. 51 (29), 7221-7225. doi:10.1002/anie.201202480

Schoop, L. M., Pielnhofer, F., and Lotsch, B. V. (2018). Chemical Principles of Topological Semimetals. Chem. Mater. 30 (10), 3155-3176. doi:10.1021/ acs.chemmater.7b05133

Sun, Q., Ma, Y., and Kioussis, N. (2020). Two-dimensional Dirac Spin-Gapless Semiconductors with Tunable Perpendicular Magnetic Anisotropy and a Robust Quantum Anomalous Hall Effect. Mater. Horiz. 7 (8), 2071-2077. doi:10.1039/d0mh00396d

Tian, L., Liu, Y., Yu, W. W., Zhang, X., and Liu, G. (2021). Triple Degenerate point in Three Dimensions: Theory and Realization. Phys. Rev. B 104 (4), 045137. doi:10.1103/physrevb.104.045137

Togo, A., and Tanaka, I. (2015). First Principles Phonon Calculations in Materials Science. Scripta Materialia 108, 1-5. doi:10.1016/j.scriptamat.2015.07.021

Wang, J. R., Li, W., and Zhang, C. J. (2020). Possible Instabilities in Quadratic and Cubic Nodal-Line Fermion Systems with Correlated Interactions. Phys. Rev. B 102 (8), 085132. doi:10.1103/physrevb.102.085132

Wang, S., and Yang, B. (2021). Dirac Nodal Line Semimetal of Three-Dimensional Cross-Linked Graphene Network as Anode Materials for Li-Ion Battery beyond Graphite. ACS Appl. Energ. Mater. 4 (3), 2091-2097. doi:10.1021/ acsaem.0c02408

Wang, X.-L. (2017). Dirac Spin-Gapless Semiconductors: Promising Platforms for Massless and Dissipationless Spintronics and New (Quantum) Anomalous Spin Hall Effects. Natl. Sci. Rev. 4 (2), 252-257. doi:10.1093/nsr/nww069 
Wang, X., Cheng, Z., Wang, J., Wang, X.-L., and Liu, G. (2016). Recent Advances in the Heusler Based Spin-Gapless Semiconductors. J. Mater. Chem. C 4 (30), 7176-7192. doi:10.1039/c6tc01343k

Wang, X., Ding, G., Cheng, Z., Surucu, G., Wang, X.-L., and Yang, T. (2020). Novel Topological Nodal Lines and Exotic Drum-head-like Surface States in Synthesized CsCl-type Binary alloy TiOs. J. Adv. Res. 22, 137-144. doi:10.1016/j.jare.2019.12.001

Wang, X., Ding, G., Cheng, Z., Surucu, G., Wang, X.-L., and Yang, T. (2020). Rich Topological Nodal Line Bulk States Together with Drum-head-like Surface States in NaAlGe with Anti-PbFCl Type Structure. J. Adv. Res. 23, 95-100. doi:10.1016/j.jare.2020.01.017

Wu, Q., Zhang, S., Song, H.-F., Troyer, M., and Soluyanov, A. A. (2018). WannierTools: An Open-Source Software Package for Novel Topological Materials. Comput. Phys. Commun. 224, 405-416. doi:10.1016/j.cpc.2017.09.033

Xie, C., Liu, Y., Zhang, Z., Zhou, F., Yang, T., Kuang, M., et al. (2021). Sixfold Degenerate Nodal-point Phonons: Symmetry Analysis and Materials Realization. Phys. Rev. B 104 (4), 045148. doi:10.1103/physrevb.104.045148

Xu, L., Zhang, X., Meng, W., He, T., Liu, Y., Dai, X., et al. (2020). Centrosymmetric TiS as a Novel Topological Electronic Material with Coexisting Type-I, Type-II and Hybrid Nodal Line States. J. Mater. Chem. C 8 (40), 14109-14116. doi:10.1039/d0tc03600e

Xu, S.-Y., Liu, C., Kushwaha, S. K., Sankar, R., Krizan, J. W., Belopolski, I., et al. (2015). Observation of Fermi Arc Surface States in a Topological Metal. Science 347 (6219), 294-298. doi:10.1126/science.1256742

Xu, Y., Elcoro, L., Song, Z.-D., Wieder, B. J., Vergniory, M. G., Regnault, N., et al. (2020). High-throughput Calculations of Magnetic Topological Materials. Nature 586 (7831), 702-707. doi:10.1038/s41586-020-2837-0

Materials Project (2021). Available at: https://materialsproject.org/materials/mp22862/ (Accessed October 23, 2021).

Yan, L., Liu, P.-F., Bo, T., Zhang, J., Tang, M.-H., Xiao, Y.-G., et al. (2019). Emergence of Superconductivity in a Dirac Nodal-Line $\mathrm{Cu}_{2} \mathrm{Si}$ Monolayer: $\mathrm{Ab}$ Initio Calculations. J. Mater. Chem. C 7 (35), 10926-10932. doi:10.1039/ c9tc03740c

Yu, Z.-M., Wu, W., Sheng, X.-L., Zhao, Y. X., and Yang, S. A. (2019). Quadratic and Cubic Nodal Lines Stabilized by Crystalline Symmetry. Phys. Rev. B 99 (12), 121106. doi:10.1103/physrevb.99.121106

Yue, Z., Li, Z., Sang, L., and Wang, X. (2020). Spin-Gapless Semiconductors. Small 16 (31), 1905155. doi:10.1002/smll.201905155
Zhang, X., Guo, R., Jin, L., Dai, X., and Liu, G. (2018). Intermetallic $\mathrm{Ca}_{3} \mathrm{~Pb}$ : a Topological Zero-Dimensional Electride Material. J. Mater. Chem. C 6 (3), 575-581. doi:10.1039/c7tc04989g

Zhao, Z., Zhang, Z., and Guo, W. (2020). A Family of All Sp ${ }^{2}$-Bonded Carbon Allotropes of Topological Semimetals with Strain-Robust Nodal-Lines. J. Mater. Chem. C 8 (5), 1548-1555. doi:10.1039/c9tc05470g

Zheng, B., Xia, B., Wang, R., Zhao, J., Chen, Z., Zhao, Y., et al. (2019). Tunable Ferromagnetic Weyl Fermions from a Hybrid Nodal Ring. npj Comput. Mater. 5 (1), 1-7. doi:10.1038/s41524-019-0214-Z

Zhong, C., Chen, Y., Xie, Y., Yang, S. A., Cohen, M. L., and Zhang, S. B. (2016). Towards Three-Dimensional Weyl-Surface Semimetals in Graphene Networks. Nanoscale 8 (13), 7232-7239. doi:10.1039/c6nr00882h

Zhou, P., Ma, Z. S., and Sun, L. Z. (2018). Coexistence of Open and Closed Type Nodal Line Topological Semimetals in Two Dimensional B2C. J. Mater. Chem. C 6 (5), 1206-1214. doi:10.1039/c7tc05095j

Zhou, P., Ma, Z. S., and Sun, L. Z. (2018). Coexistence of Open and Closed Type Nodal Line Topological Semimetals in Two Dimensional $\mathrm{B}_{2}$ C. J. Mater. Chem. C 6 (5), 1206-1214. doi:10.1039/c7tc05095j

Zhu, Z., Winkler, G. W., Wu, Q., Li, J., and Soluyanov, A. A. (2016). Triple point Topological Metals. Phys. Rev. X 6 (3), 031003. doi:10.1103/physrevx.6.031003

Conflict of Interest: The authors declare that the research was conducted in the absence of any commercial or financial relationships that could be construed as a potential conflict of interest.

Publisher's Note: All claims expressed in this article are solely those of the authors and do not necessarily represent those of their affiliated organizations, or those of the publisher, the editors and the reviewers. Any product that may be evaluated in this article, or claim that may be made by its manufacturer, is not guaranteed or endorsed by the publisher.

Copyright (C) 2021 Zhang, Fang, Cheng, Lin and Wang. This is an open-access article distributed under the terms of the Creative Commons Attribution License (CC BY). The use, distribution or reproduction in other forums is permitted, provided the original author(s) and the copyright owner(s) are credited and that the original publication in this journal is cited, in accordance with accepted academic practice. No use, distribution or reproduction is permitted which does not comply with these terms. 